\title{
REVIEW
}

\section{COVID-19 and cardiovascular disease}

\author{
Pawan Suri ${ }^{1}$ and Vanita Arora ${ }^{2, *}$ \\ ${ }^{1}$ SGL Superspeciality Hospital, Jalandhar, Punjab-144022, India \\ ${ }^{2}$ Cardiac Electrophysiology Laboratory \& Arrhythmia Services, Max Healthcare Superspeciality Hospital, Saket, New Delhi-110017, India
}

\begin{abstract}
Coronavirus disease 2019 (COVID-19), a spectrum of disease spanning from a barely symptomatic infection to critical illness, is caused by the new coronavirus - severe acute respiratory syndrome coronavirus 2 (SARS-CoV-2). Although lung injury and acute respiratory distress syndrome have been at a centre stage as the most dreadful complications of COVID-19, the heart damage has recently emerged as yet another grim outcome in the virus's repertoire of possible complications. The cardiac involvement occurs as a direct invasion of cardiomyocytes by the virus via the angiotensin-converting enzyme 2 (ACE2) receptors and also indirectly by the inflammatory reaction due to cytokine storm. COVID-19 increases the severity of an already pre-existing heart disease and also unmasks the cardiac symptoms in a previously undiagnosed heart disease as well. Apart from this the drugs that are repurposed and are supposed to have a preventive or a therapeutic potential are also supposedly causing cardiac side effects. In the absence of substantial database all these seem to be a hypothetical correlation and one needs to rely on clinical skills and acumen.
\end{abstract}

Keywords: COVID-19; SARS-CoV-2; cardiovascular disease; acute respiratory distress syndrome

*Corresponding author: Dr. Vanita Arora, MD; DNB (Cardiology); FRCP (Edin)., Director \& Head, Cardiac Electrophysiology lab \& Arrhythmia Services, Max Healthcare Superspeciality Hospital, Saket, New Delhi-110017, India. Email: aroraheartrhythmdoc@ gmail.com

Received 15 September 2020; Revised 30 October 2020; Accepted 14 November 2020; Published 24 November 2020

Citation: Suri P, Arora V. COVID-19 and cardiovascular disease. J Med Sci Res. 2020; 8(S1):69-73. DOI: http://dx.doi.org/10.17727/ JMSR.2020/8S1-8

Copyright: (c) 2020 Suri P et al. Published by KIMS Foundation and Research Center. This is an open-access article distributed under the terms of the Creative Commons Attribution License, which permits unrestricted use, distribution, and reproduction in any medium, provided the original author and source are credited.

\section{Introduction}

Coronavirus disease 2019 (COVID-19) that originated in Wuhan, China in late December 2019, is an unprecedented health crisis that does not differentiate between borders, ethnicities, age, gender or disability status and in a time duration of few months only has become a 'Public Health Emergency of International Concern' [1] As known to all, the health impacts of this virus are devastating and, rightly, in the forefront of our minds, the broken and undermined healthcare system of each and every country has got exposed. Both the lives and livelihood of millions of people have been affected and lakhs have lost their loved ones. While it continues to spread it is important that communities take action to prevent further transmission, reduce the impacts of the outbreak and support control measures. 
At present, preventive vaccines and prophylactic therapies for COVID-19 are not available.

COVID-19 is caused by a severe acute respiratory syndrome coronavirus 2 (SARS-CoV-2), which has a $79.6 \%$ genomic sequence identity with SARS$\mathrm{CoV}$ and shares the many biological features of the same [2]. Although it causes the respiratory infection (pneumonia/ARDS) and in uncontrolled situations a cytokine storm, early clinical data is suggestive of a strong association between the COVID-19 and cardiovascular disease. Furthermore, COVID-19 seems to promote the development of cardiovascular disorders, such as myocardial injury, arrhythmias, acute coronary syndrome (ACS) and venous thromboembolism owing to coagulation abnormalities.

In this review, we summarize our current knowledge of SARS-CoV-2, its clinical effects on cardiovascular system, link between cardiovascular drugs and COVID-19 and the potential cardiovascular effects of drugs used to treat COVID-19.

\section{SARS-CoV-2}

Corona viruses are divided into four genera (alpha, beta, gamma, delta), of which $\alpha$ and $\beta$-corona viruses are known to infect humans. SARS-CoV-2, the 7th known human corona virus, is a member of the family beta corona virus and is similar to the zoonotic severe acute respiratory syndrome (SARS) coronavirus (SARS-CoV) from 2002 and the Middle East respiratory syndrome (MERS) coronavirus (MERS-CoV) from 2012. The knowledge about the biology and pathogenesis of the latter over the last decade has made it possible for us to understand the many biological features of SARS-CoV-2 as there is a genomic sequence homology of $79.6 \%$.

Both the SARS-CoV and SARS-CoV-2 have four structural proteins $(\mathrm{S}, \mathrm{E}, \mathrm{M}, \mathrm{N})$ with the $\mathrm{S}$ protein playing a pivotal role in virus attachment and entry followed by disease progression [3]. However, the S protein in SARS-CoV-2 has a novel furin cleavage site that makes it more pathogenic and capable of causing multi organ damage and has higher affinity to human angiotensin-converting enzyme 2 (ACE2) receptor resulting in higher transmissibility and severity of COVID-19. Once the virus gets attached to the ACE2 receptor, the viral complex is incorporated into the cytoplasm either by direct fusion or via endocytosis and finally followed by the RNA replication by RNA dependent RNA polymerase.

\section{ACE2 and tissue tropism}

SARS-CoV-2 preferentially infects the respiratory tract and the systemic inflammation induced by viral pneumonia leads to involvement of other organ systems as well. However, the presence of virus particles on histological examination suggests the direct viral infection in non-respiratory organs such as heart, kidney, liver and brain. The cardiovascular manifestations are an outcome of both the systemic inflammation and the direct involvement of cardiomyocytes.

ACE2 is highly expressed at the tissue level in lungs, kidneys, heart, blood vessels and in some studies the expression of ACE2 is even higher in heart and coronary vessels than in lungs. The binding of viral $S$ protein to ACE2 in cardiomyocytes triggers the activation of TMPRSS2 that facilitates S protein priming and promotes entry of the virus into the cell. However, in patients with low expression of ACE2 in cardiomyocytes and pericytes cathepsin $\mathrm{B}$ and cathepsin $\mathrm{L}$ is activated and virus entry into cell is facilitated via the endocytic pathway. The viral genomic RNA is then replicated by RdRP and finally the genomic RNA and structural proteins are assembled into new viral particles, leading to their release via exocytosis [4].

SARS-CoV-2 infection causes a marked downregulation of ACE2 that leads to reduction in cardiac contractility, greater atherosclerotic plaque accumulation and upgradation of adhesion molecules and inflammatory cytokines.

\section{The cardiovascular system and COVID-19}

COVID-19 causes no or mild symptoms in a significant majority (81\%) of patients, substantial symptoms like dyspnea, respiratory rate $\geq 30 / \mathrm{min}$, blood oxygen saturation $\leq 93 \%$ in $14 \%$ and severe symptoms like respiratory failure, septic shock, or multiple organ dysfunction or failure in $5 \%$ of critically ill patients [5]. The overall case fatality rate on an average is between $2-3 \%$ but an individual case fatality rate is highest among those with associated cardiovascular disease $(10.5 \%)$ and associated comorbidities like hypertension $(6 \%)$ and diabetes $(7.3 \%)$ and obesity. 
Although the predominant clinical manifestation of COVID-19 is viral pneumonia, COVID-19 can also cause cardiovascular disorders such as myocardial injury, arrhythmias, ACS and thromboembolism. Cardiac symptoms can be the first clinical manifestation in those patients who present without the typical symptoms of fever, cough etc.

\section{Myocarditis}

Acute myocardial injury, evidenced by elevated levels of cardiac biomarkers (hS Trop I, hS CRP) or electrocardiographic abnormalities is an independent risk factor associated with a significantly worse prognosis [6]. It results from the direct viral infection of heart or in response to the systemic inflammation due to cytokine storm. However, in majority of patients, the typical signs of myocarditis like segmental wall motion abnormality, reduced left ventricular ejection fraction (LVEF) or mild pericardial effusion may not be seen.

\section{Acute coronary syndrome}

COVID-19 has the potential to cause ACS and although the actual incidence is unknown, there are reports that COVID-19 can cause ACS with or without the presence of systemic inflammation. The plaque rupture, coronary spasm or microthrombi are the proposed mechanisms. The plaque rupture occurs due to the degradation of collagen, a major constituent of fibrous cap, by collagenases secreted from activated macrophages and the microthrombi resulting in thrombus formation is potentiated by the tissue factor [7]. The direct endothelial or vascular injury caused by SARS-CoV-2 infection might also increase the risk of thrombus formation and ACS.

\section{Heart failure}

Approximately, one fourth of patients admitted with COVID-19 develop the clinical features of heartfailure, evidenced by elevated levels of amino-terminal proB-type natriuretic peptide [8]. As patients with COVID-19 have pre-existing comorbidities such as coronary artery disease, hypertension and diabetes, heart failure might be the result of an exacerbation of these pre-existing conditions or a new onset myocardial injury either due to myocarditis or ACS. In addition the myocardial dysfunction related to cytokine storm, as also high fever, tachycardia, impaired renal function, triggers the development of heart failure in patients with reduced or preserved ejection fraction.

\section{Cardiac arrhythmias}

COVID-19 has an uncertain role in the development of cardiac arrhythmias [9]. However, all types of atrial and ventricular arrhythmias ranging from brady to tachy arrhythmias have been seen in COVID19 patients either related to the direct myocardial injury or to the systemic causes such as fever, hypoxia, electrolyte abnormalities and sepsis. The usage of antiviral medications and few antibiotics in treatment of COVID-19 are also known to induce arrhythmias in few patients.

\section{Coagulation abnormalities}

COVID-19 is associated with coagulation abnormalities that can lead to both the venous and arterial thromboembolism. An elevated levels of D-dimer, fibrinogen, factor VIII, modestly reduced platelet counts and slightly prolonged prothrombin time due to severe inflammatory response and endothelial damage in combination with underlying comorbidities is seen in these patients. In addition, certain antiviral medications and other therapies used in these patients are the potential contributors to thrombus formation [10].

\section{Cardiac biomarkers and prognostication}

Cardiac specific biomarkers (CK, CK-MB, Troponin, Myoglobin, and BNP) are important in recognizing patients of COVID-19 with early cardiac involvement as the presence of myocardial injury has been associated with over $50 \%$ mortality. The increased troponin level is not only an independent high risk factor but is also associated with elevation of other acute phase reactants. The elevation in troponin is seen in almost $12 \%$ of COVID-19 patients and is believed to reflect a non-coronary disease due to decrease in oxygen availability compared to an increased need in presence of cytokine storm in critically unwell COVID-19 patients. However, it is generally accepted that troponin should only be measured if AMI is being suspected. The elevated troponin also has a potential prognostic value as exemplified by Guo et al, that revealed that elevated troponin level was associated with a mortality of $59.6 \%$ compared to $8.9 \%$ in patients with normal levels. High mortality was seen in patients with elevated levels even without a history of CVD (37.5\% vs $69.4 \%$ ).

\section{Drugs and cardiovascular disease}

There is neither any treatment nor a vaccine 
available for COVID-19 as of now. Since it has a genomic resemblance with SARS and MERS, the medications which have been used and are effective against these viruses have been repurposed for usage in the current epidemic with controversial results [11]. However, the earliest controversy at the origin of COVID-19 that got highlighted was the effect of ACE-I on COVID-19 disease susceptibility and progression.

\section{ACE-I's and COVID-19}

The ACE2 is a receptor for SARS-CoV-2, hence clinicians expressed concern that medications that upregulate the cell surface expression of ACE2 might be harmful as ACE I's and ARBs have been shown to increase the expression of ACE2 in animal models. Both ACE I's and ARBs are commonly used worldwide for the treatment of HT and other CVDs, hence the deliman of these drugs to be discontinued during the COVID-19 pandemic became a pertinent question. The indiscriminate withdrawal of these drugs could harm high-risk patients. Henceforth, the American College of Cardiology/ American Heart Association (ACC/AHA), the European Society of Cardiology (ESC) and the Heart Failure Society of America have issued statements recommending continuation of renin-angiotensin-aldosterone system (RAAS) antagonists for those who are currently prescribed these agents [12]. It is supported by the findings from clinical studies from Spain $(n=1,139)$, Italy $(n=6,772)$ and the USA ( $n=5,894$ and 1,735), in which the use of RAAS inhibitors was not associated with a positive COVID-19 test, suggesting that these agents do not affect susceptibility to SARS-CoV-2 infection. Furthermore, the use of these agents was not associated with a substantial increase in the risk of severe or fatal illness among patients with COVID19.

Hydroxychloroquine blocks the entry of virus into host cells by inhibiting the endosomal pathway and also attenuates the cytokine production. In combination with azithromycin there occurs an increase in QT interval and the likelihood of cardiac arrest also increases markedly [13]. Given that some patients with COVID-19 might have impaired renal function owing to systemic illness, frequent electrocardiographic evaluation should be strongly considered in patients treated with hydroxchloroquine and/or azithromycin.
Remdesivir has been used for the treatment of Ebola virus diseaseand as prophylaxis in MERS to reduce the viral load [14]. Although prominent cardiovascular adverse effects associated with remdesivir have not been reported so far, these might become apparent with more widespread use.

Lopinavir-ritonavir inhibits the protease activity and is used for the prevention and treatment of HIV infection. Since the drug combination is metabolised by cytochrome P450 3A4, it should be used cautiously to avoid interaction with the commonly used cardiovascular drugs including antiplatelet agents, antiarrhythmic agents and anticoagulants [15].

Tocilizumab is one of the IL-6 receptor blocking agents proposed for treatment of COVID19 , has been used in the early 2000's for treatment of autoimmune disorders such as refractory rheumatoid arthritis and systemicjuvenile idiopathic arthritis (sJIA) and was approved by the FDA in 2017 for treatment of the cytokine release syndrome that may occur following some forms of immunotherapy. In severe cases of COVID-19, there is an increase in cytokines, among which IL-6 especially Tocilizumab seems to play an important role. 19 studies were analysed, including 11 RCTs and 8 cohort studies and despite an increase in cholesterol levels, tocilizumab (TCZ) had safe cardiovascular outcomes.

\section{Post COVID-19 recovery syndrome}

Based on the data of patients with recovered myocarditis, MI, or other cardiac injury, it is expected that some patients will have subclinical and possibly overt cardiovascular abnormalities. There have been reports of delayed manifestations weeks or months after recovery in form of thromboembolism, arrhythmias, acute coronary syndrome and more drastically sudden cardiac arrest. In addition, patients who have recovered from acute stage also seem to be at high risk of recurrent events. While current treatment appropriately focuses on acute recovery, it is unknown whether the treatment given during the acute illness may affect future CV abnormalities. Keeping in view the long-term impact of COVID-19 and cytokine storm, it is imperative to determine if acute delivery of antifibrotic therapy, anti-inflammatory therapy, cell-based therapy, or 
antiviral therapy affects long-term as well as shortterm outcomes [16].

\section{Conclusion}

The SARS-CoV-2 causing COVID-19 reached pandemic levels in March 2020. In the absence of vaccines or curative medical treatment, COVID-19 exerts an unprecedented global impact on public health and health care delivery. Apart from causing viral pneumonia SARS-CoV-2 has major implications for the CV system. Patients with CV risk factors including male sex, advanced age, diabetes, hypertension and obesity, already established CV and cerebrovascular disease have been identified as particularly vulnerable populations with increased morbidity and mortality when suffering from COVID-19.

Moreover, a considerable proportion of patients may develop cardiac injury in the context of COVID19 which increases risk of in-hospital mortality. Aside from arterial and venous thrombotic complications presenting as acute ACS and VTE, myocarditis plays an important role in patients with acute HF. A wide range of arrhythmias have been reported to complicate the course of COVID-19 including potential pro-arrhythmic effects of medical treatment targeted at COVID-19 and associated diseases which is further aggravated by increasing concerns of delayed presentation of CV emergencies as patients are afraid to seek medical attention during the pandemic.

\section{Conflicts of interest}

Authors declare no conflicts of interest.

\section{References}

[1] Wu F, Zhao S, Yu B, Chen YM, Wang W, et al. A new coronavirus associated with human respiratory disease in China. Nature. 2020; 579(7798):265-269.

[2] Lu R, Zhao X, Li J, Niu P, Yang B, et al. Genomic characterisation and epidemiology of 2019 novel coronavirus: implications for virus origins and receptor binding. Lancet. 2020; 395(10224):565-574.

[3] Walls AC, Park YJ, Tortorici MA, Wall A, McGuire AT, et al. Structure, function, and antigenicity of the SARS-CoV-2 spike glycoprotein. Cell. 2020; 181(2):281-292.

[4] Hoffmann M, Kleine-Weber H, Schroeder S, Krüger N, Herrler T, et al. SARS-CoV-2 cell entry depends on ACE2 and TMPRSS2 and is blocked by a clinically proven protease inhibitor. Cell. 2020; 181(2):271-280.

[5] Clerkin KJ, Fried JA, Raikhelkar J, Sayer G, Griffin JM, et al. COVID-19 and cardiovascular disease. Circulation. 2020; 141(20):1648-1655.
[6] Shi S, Qin M, Cai Y, Liu T, Shen B, et al. Characteristics and clinical significance of myocardial injury in patients with severe coronavirus disease 2019. Eur Heart J. 2020; 41(22):2070-2079.

[7] Stefanini GG, Montorfano M, Trabattoni D, Andreini D, Ferrante G, et al. ST-elevation myocardial infarction in patients with COVID-19: clinical and angiographic outcomes. Circulation. 2020; 141(25):2113-2116.

[8] Mehra MR, Ruschitzka F. COVID-19 illness and heart failure: a missing link? JACC Heart Fail. 2020; 8(6):512-514.

[9] Lakkireddy DR, Chung MK, Gopinathannair R, Patton KK, Gluckman TJ, et al. Guidance for cardiac electrophysiology during the COVID-19 pandemic from the Heart Rhythm Society COVID-19 task force; electrophysiology section of the American College of Cardiology; and the electrocardiography and arrhythmias committee of the council on clinical cardiology, American Heart Association. Circulation. 2020; 141(21):e823-e831.

[10] Connors JM, Levy JH. Thromboinflammation and the hypercoagulability of COVID-19. J Thromb Haemost. 2020; 18(7):1559-1561.

[11] Chinese Society of Cardiology. Scientific statement on using renin-angiotensin system blockers in patients with cardiovascular disease and COVID-19. Chin J Cardio. 2020; 48(0):E014.

[12] Pushpakom S, Iorio F, Eyers PA, Escott KJ, Hopper S, et al. Drug repurposing: progress, challenges and recommendations. Nat Rev Drug Discov. 2019; 18(1):41-58.

[13] Geleris J,Sun Y, Platt J, Zucker J, Baldwin M, et al. Observational study of hydroxychloroquine in hospitalized patients with COVID-19. N Engl J Med. 2020; 382(25):2411-2418.

[14] Wang Y, Zhang D, Du G, Du R, Zhao J, et al. Remdesivir in adults with severe COVID-19: a randomised, doubleblind, placebo-controlled, multicentre trial. Lancet. 2020; 395(10236):1569-1578.

[15] Sheahan TP, Sims AC, Leist SR, Schäfer A, Won J, et al. Comparative therapeutic efficacy of remdesivir and combination lopinavir, ritonavir, and interferon beta against MERS-CoV. Nat Commun. 2020; 11(1):222.

[16] Shafi AMA, Shaikh SA, Shirke MM, Iddawela S, Harky A. Cardiac manifestations in COVID-19 patients-A systematic review. J Card Surg. 2020; 35(8):1988-2008. 